\title{
Synthesis and Characterization of Some Benzylidinehydrazinyl Derivatives of Newer Pyrimidine-5-carbonitrile Moiety
}

\author{
Hingrajia Dhaval, Thanki Pragna, Modha Jayesh* \\ Department of Chemistry, Maharshi Dayanand Science College, Porbandar, Gujarat, India
}

*E-mail address: drjmodha@gmail.com

\begin{abstract}
Condensation of 2-hydrazinyl-1,6-dihydro-1-methyl-6-oxo-4-isobutylpyrimidine-5-carbonitrile (3) with different aromatic aldehyde gave the corresponding 2-(Benzylidinehydrazinyl)-1,6-dihydro-6oxo-4-isobutylpyrimidine-5-carbonitrile (4). The reaction between 1,6-dihydro-1-methyl-2(methylthio)-6-oxo-4-isobutylpyrimidine-5-carbonitrile (2) with hydrazine hydrate furnished (3). The condensation of 1,2,3,4-tetrahydro-4-oxo-6-isobutyl-2-thioxopyrimidine-5-carbonitrile (1) with methyl iodide yielded 2. Finally the products were characterized by ${ }^{1} \mathrm{H}$ NMR, Mass and IR Spectra.
\end{abstract}

Keyword: Cyanopyrimidine; Benzylidinehydrazinyl; Isovaleraldehyde

\section{INTRODUCTION}

Schiff bases are an important class of organic compounds. They were first prepared by German chemist Hugo Schiff [1]. These compounds are prepared by condensation of primary amine with compound containing an active carbonyl group \& elimination of water molecule. The structural feature of these compounds are the azomethine group having the general formula $\mathrm{RHC}=\mathrm{N}-\mathrm{R}_{1}$, where $\mathrm{R}$ and $\mathrm{R}_{1}$ are alkyl, aryl, cycloalkyl, or heterocyclic groups. They are known to exhibit a broad range of biological activities including antifungal, antibacterial, antimalarial, anti-inflammatory, antiviral, and antipyretic properties [2,3]. Imine or azomethine group present in various natural and synthetic compounds has been found to be critical to their biological activities [4-6]. Schiff's bases are important compounds also owing to their wide range of industrial applications [7]. They are well known intermediates for synthesis of pharmacologically active heterocycles like oxadiazolines, imidazolinones, azetidinones, thiazolidinones [8] and many other derivatives.

1,2,3,4-tetrahydropyrimidine-5-carbonitrile scaffold [9] have demonstrated biological activities like Calcium channel antagonist [10], Cardiovascular [11], Anti-inflammatory [12], Antimicrobial [13-15], and Immunomodulatory [16].

Schiff base associated with Pyrimidine-5-carbonitrile have also been reported to exhibit a wide range of biological activities like Antitubercular, Anticonvulsant, Neurotoxicity [17], Antitumor [18] and Antibacterial [19].

Going through the references and in search of newer pharmacologically active pyrimidine-5-carbonitrile derivatives, we have synthesized some new 2(Benzylidinehydrazinyl)-1,6-dihydro-6-oxo-4-isobutylpyrimidine-5-carbonitrile by condensation of 2-hydrazinyl-1,6-dihydro-1-methyl-6-oxo-4-isobutylpyrimidine-5- 
carbonitrile with different aromatic aldehydes using 3-component heterocyclization method $[20]$.

\section{EXPERIMENTAL}

Melting points were taken in open capillary and are not corrected. Purity of synthesized compounds have been checked by TLC. ${ }^{1} \mathrm{H}$ NMR spectra were recorded in Bruker-Avance-II (400 MHz) using DMSO-d6 as a solvent and TMS as an internal standard and the chemical shifts are reported as parts per million ( $\mathrm{ppm}$ ). Mass spectra were determined on ShimadzuQP2010 spectrometer. IR spectra were recorded on Shimadzu-FTIR-8400 using KBr pallet.<smiles>CCOC(=O)COc1nc(CC(C)C)c(C)c(=O)n1C</smiles>

Scheme 1

\section{1. Synthesis of 1,2,3,4-tetrahydro-6-isobutyl-4-oxo-2-thioxopyrimidine-5-carbonitrile}

(1)

A mixture of Thiourea $(0.05 \mathrm{~mol})$, ethylcyanoacetate $(0.05 \mathrm{~mol})$, isovaleraldehyde $(0.05$ $\mathrm{mol})$ and potassium carbonate $(0.05 \mathrm{~mol})$ in absolute alcohol $(100 \mathrm{ml})$ was refluxed for 4 hours. Reaction mixture was poured into minimum quantity of crushed ice and neutralized with acetic acid. The product obtained was isolated and crystallized from water. Mass $\mathrm{M}^{+}=$ 209: IR (KBr) v (cm $\left.{ }^{-1}\right), 2965$ (-CH $\mathrm{CH}_{3}$ Asym.), $2877\left(-\mathrm{CH}_{3}, \mathrm{Sym}.\right), 2235$ (-CN), 1648 (-CO); $1 \mathrm{H}$ NMR $(\delta \mathrm{ppm})(400 \mathrm{MHz}, \mathrm{DMSO}), \delta 0.9\left(\mathrm{~d}, 6 \mathrm{H}, \mathrm{CH}_{3}\right), \delta 2.0(\mathrm{~m}, 1 \mathrm{H}, \mathrm{CH}), \delta 2.4(\mathrm{~d}, 2 \mathrm{H}$, $\left.\mathrm{CH}_{2}\right), \delta 13(\mathrm{~s}, 2 \mathrm{H}, \mathrm{NH})$. 


\section{2. Synthesis of 1,6-dihydro-4-isobutyl-1-methyl-2-(methylthio)-6-oxopyrimidine-5- carbonitrile (2)}

To a solution of (1) $(0.05 \mathrm{~mol})$ in DMF $(70 \mathrm{ml})$, potassium carbonate $(0.1 \mathrm{~mol})$ and methyliodide $(0.1 \mathrm{~mol})$ were added and the mixture was stirred for 3 hours. The contents were poured into water, filtered, washed with water and crystallized from DMF. Mass $\mathrm{M}^{+}=237$ : IR

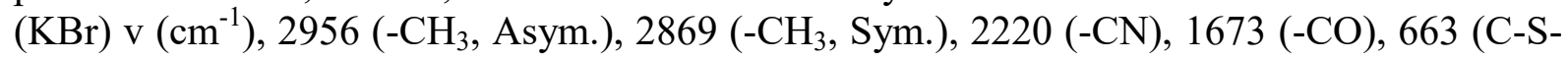
C); $1 \mathrm{H} \mathrm{NMR}(\delta \mathrm{ppm})(400 \mathrm{MHz}, \mathrm{DMSO}), \delta 0.9\left(\mathrm{~d}, 6 \mathrm{H}, \mathrm{CH}_{3}\right), \delta 2.1(\mathrm{~m}, 1 \mathrm{H}, \mathrm{CH}), \delta 2.5(\mathrm{~d}$, $\left.2 \mathrm{H}, \mathrm{CH}_{2}\right), \delta 2.6\left(\mathrm{~s}, 3 \mathrm{H}, \mathrm{S}-\mathrm{CH}_{3}\right), \delta 3.4\left(\mathrm{~s}, 3 \mathrm{H}, \mathrm{N}-\mathrm{CH}_{3}\right)$.

\section{3. Synthesis of 2-hydrazinyl-1,6-dihydro-4-isobutyl-1-methyl-6-oxopyrimidine-5- carbonitrile (3)}

A mixture of (2) $(0.01 \mathrm{~mol})$ and Hydrazine hydrate $(3.5 \mathrm{ml})$ in absolute alcohol $(30 \mathrm{ml})$ was refluxed for 6 hours. The reaction mixture was poured into crushed ice and the solid product obtained after neutralization with acetic acid was kept in water overnight. The product was isolated and crystallized from absolute alcohol. Mass $\mathrm{M}^{+}=221$ : $\mathrm{IR}(\mathrm{KBr}) \mathrm{v}\left(\mathrm{cm}^{-}\right.$ $\left.{ }^{1}\right), 3304$ (-NH, secondary) 2954 (-CH3, Asym.), 2868 (-CH $\mathrm{CH}_{3}$, Sym.), 2220 (-CN), 1676 (-CO); $1 \mathrm{H}$ NMR $(\delta \mathrm{ppm})(400 \mathrm{MHz}, \mathrm{DMSO}), \delta 0.9\left(\mathrm{~d}, 6 \mathrm{H}, \mathrm{CH}_{3}\right), \delta 2.2(\mathrm{~m}, 1 \mathrm{H}, \mathrm{CH}), \delta 2.3(\mathrm{~d}, 2 \mathrm{H}$, $\mathrm{CH} 2), \delta 3.1\left(\mathrm{~s}, 3 \mathrm{H}, \mathrm{N}-\mathrm{CH}_{3}\right)$.

\section{4. Synthesis of 2-(Substituted benzylidenehydrazinyl)-1,6-dihydro-4-isobutyl-1- methyl-6-oxopyrimidine-5-carbonitrile (4a-j)}

A mixture of (3) $(0.01 \mathrm{~mol})$ and different aromatic aldehyde $(0.01 \mathrm{~mol})$ in absolute alcohol $(20 \mathrm{ml})$ was refluxed for 3 hours in presence of catalytic amount of acetic acid. The reaction mixture was poured into sodium bisulfite solution. The product was isolated and crystalized from appropriate solvent.

Table 1. Physical constant.

\begin{tabular}{|c|c|c|c|c|c|c|c|}
\hline Comp. & $\mathrm{R}$ & $\mathrm{M} . \mathrm{F}$. & $\begin{array}{c}\mathrm{MP} \\
{ }^{\circ} \mathrm{C}\end{array}$ & $\begin{array}{c}\text { Yield } \\
\%\end{array}$ & $\begin{array}{c}\text { \% of } \mathrm{C} \\
\text { Found } \\
(\text { Calcd.) }\end{array}$ & $\begin{array}{c}\text { \% of } \mathrm{H} \\
\text { Found } \\
\text { (Calcd.) }\end{array}$ & $\begin{array}{c}\text { \% of } \mathrm{N} \\
\text { Found } \\
\text { (Calcd.) }\end{array}$ \\
\hline 1 & - & $\mathrm{C}_{9} \mathrm{H}_{11} \mathrm{~N}_{3} \mathrm{OS}$ & 246 & 90 & $\begin{array}{c}51.63 \\
(51.65)\end{array}$ & $\begin{array}{c}5.27 \\
(5.30)\end{array}$ & $\begin{array}{c}20.05 \\
(20.08)\end{array}$ \\
\hline 2 & - & $\mathrm{C}_{11} \mathrm{H}_{15} \mathrm{~N}_{3} \mathrm{OS}$ & 100 & 85 & $\begin{array}{c}55.32 \\
(55.37)\end{array}$ & $\begin{array}{c}6.34 \\
(6.37)\end{array}$ & $\begin{array}{c}17.68 \\
(17.71)\end{array}$ \\
\hline 3 & - & $\mathrm{C}_{10} \mathrm{H}_{15} \mathrm{~N}_{5} \mathrm{O}$ & 168 & 60 & $\begin{array}{c}54.25 \\
(54.28)\end{array}$ & $\begin{array}{c}6.79 \\
(6.83)\end{array}$ & $\begin{array}{c}31.61 \\
(31.65)\end{array}$ \\
\hline $4 \mathrm{a}$ & $-\mathrm{H}$ & $\mathrm{C}_{17} \mathrm{H}_{19} \mathrm{~N}_{5} \mathrm{O}$ & 200 & 54 & $\begin{array}{c}65.97 \\
(66.00)\end{array}$ & $\begin{array}{c}6.15 \\
(6.19)\end{array}$ & $\begin{array}{c}22.65 \\
(22.64)\end{array}$ \\
\hline $4 \mathrm{~b}$ & $-4-\mathrm{Cl}$ & $\mathrm{C}_{17} \mathrm{H}_{18} \mathrm{~N}_{5} \mathrm{OCl}$ & 206 & 55 & $\begin{array}{c}59.36 \\
(59.39)\end{array}$ & $\begin{array}{c}5.23 \\
(5.28)\end{array}$ & $\begin{array}{c}20.35 \\
(20.37)\end{array}$ \\
\hline $4 \mathrm{c}$ & $-4-\mathrm{OCH} 3$ & $\mathrm{C}_{18} \mathrm{H}_{21} \mathrm{~N}_{5} \mathrm{O}_{2}$ & 190 & 48 & $\begin{array}{c}63.65 \\
(63.70)\end{array}$ & $\begin{array}{c}6.20 \\
(6.24)\end{array}$ & $\begin{array}{c}20.59 \\
(20.64)\end{array}$ \\
\hline $4 \mathrm{~d}$ & $-4-\mathrm{N}\left(\mathrm{CH}_{3}\right)_{2}$ & $\mathrm{C}_{19} \mathrm{H}_{24} \mathrm{~N}_{6} \mathrm{O}$ & 212 & 53 & $\begin{array}{c}64.76 \\
(64.75)\end{array}$ & $\begin{array}{c}6.87 \\
(6.86)\end{array}$ & $\begin{array}{c}23.83 \\
(23.85)\end{array}$ \\
\hline $4 \mathrm{e}$ & $-3,4\left(\mathrm{OCH}_{3}\right)_{2}$ & $\mathrm{C}_{19} \mathrm{H}_{23} \mathrm{~N}_{5} \mathrm{O}_{3}$ & 186 & 56 & $\begin{array}{c}61.74 \\
(61.77)\end{array}$ & $\begin{array}{c}6.24 \\
(6.28)\end{array}$ & $\begin{array}{c}18.96 \\
(18.96)\end{array}$ \\
\hline $4 \mathrm{f}$ & $-3-\mathrm{OCH}{ }_{3}-4-$ & $\mathrm{C}_{18} \mathrm{H}_{21} \mathrm{~N}_{5} \mathrm{O}_{3}$ & 206 & 49 & $\begin{array}{c}60.82 \\
(60.83)\end{array}$ & $\begin{array}{c}5.94 \\
(5.96)\end{array}$ & $\begin{array}{c}19.68 \\
(19.71)\end{array}$ \\
\hline
\end{tabular}




\begin{tabular}{|c|c|c|c|c|c|c|c|}
\hline $4 \mathrm{~g}$ & $-4-\mathrm{CH}_{3}$ & $\mathrm{C}_{18} \mathrm{H}_{21} \mathrm{~N}_{5} \mathrm{O}$ & 190 & 52 & $\begin{array}{c}66.86 \\
(66.85)\end{array}$ & $\begin{array}{c}6.56 \\
(6.55)\end{array}$ & $\begin{array}{c}21.63 \\
(21.66)\end{array}$ \\
\hline $4 \mathrm{~h}$ & $-4-\mathrm{F}$ & $\mathrm{C}_{17} \mathrm{H}_{18} \mathrm{FN}_{5} \mathrm{O}$ & 170 & 58 & $\begin{array}{c}62.35 \\
(62.37)\end{array}$ & $\begin{array}{c}5.51 \\
(5.54)\end{array}$ & $\begin{array}{c}21.40 \\
(21.39)\end{array}$ \\
\hline $4 \mathrm{i}$ & $-4-\mathrm{OH}$ & $\mathrm{C}_{17} \mathrm{H}_{19} \mathrm{~N}_{5} \mathrm{O}_{2}$ & 242 & 60 & $\begin{array}{c}62.71 \\
(62.75)\end{array}$ & $\begin{array}{c}5.88 \\
(5.89)\end{array}$ & $\begin{array}{c}21.50 \\
(21.52)\end{array}$ \\
\hline $4 \mathrm{j}$ & $-2-\mathrm{Cl}$ & $\mathrm{C}_{17} \mathrm{H}_{18} \mathrm{~N}_{5} \mathrm{OCl}$ & 184 & 54 & $\begin{array}{c}59.38 \\
(59.39)\end{array}$ & $\begin{array}{c}5.26 \\
(5.28)\end{array}$ & $\begin{array}{c}20.32 \\
(20.37)\end{array}$ \\
\hline
\end{tabular}

\section{SPECTRAL ANALYSIS OF NOVEL BENZYLIDINEHYDRAZINYL DERIVATIVES}

\section{1. 2-(Benzylidenehydrazinyl)-1,6-dihydro-4-isobutyl-1-methyl-6-oxopyrimidine-5- carbonitrile (4a)}

Mass $\mathrm{M}^{+}=309$ : IR $(\mathrm{KBr}) \mathrm{v}\left(\mathrm{cm}^{-1}\right), 2966\left(-\mathrm{CH}_{3}\right.$, Asym.), $2872\left(-\mathrm{CH}_{3}, \mathrm{Sym}.\right), 2222$ ($\mathrm{CN}), 1668(-\mathrm{CO}), 1496-1458(\mathrm{C}=\mathrm{C}), 1084(\mathrm{~N}-\mathrm{C}) ; 1 \mathrm{H}$ NMR $(\delta \mathrm{ppm})(400 \mathrm{MHz}, \mathrm{DMSO}), \delta$ $1.0\left(\mathrm{~d}, 6 \mathrm{H}, \mathrm{CH}_{3}\right), \delta 2.0(\mathrm{~m}, 1 \mathrm{H}, \mathrm{CH}), \delta 2.7\left(\mathrm{~d}, 2 \mathrm{H}, \mathrm{CH}_{2}\right), \delta 3.2\left(\mathrm{~s}, 3 \mathrm{H}, \mathrm{N}-\mathrm{CH}_{3}\right), \delta$ 7.4-8.4 (m, $5 \mathrm{H}, \mathrm{Ar}-\mathrm{H}), \delta 10.9(\mathrm{~s}, 1 \mathrm{H}, \mathrm{NH})$.

\section{2. 2-(4-Chloro benzylidenehydrazinyl)-1,6-dihydro-4-isobutyl-1-methyl-6- oxopyrimidine-5-carbonitrile (4b)}

Mass $\mathrm{M}^{+}=343$ : IR $(\mathrm{KBr})$ v $\left(\mathrm{cm}^{-1}\right), 2962\left(-\mathrm{CH}_{3}\right.$, Asym. $), 2875\left(-\mathrm{CH}_{3}, \mathrm{Sym}.\right), 2224$ ($\mathrm{CN}), 1661(-\mathrm{CO}) ; 1 \mathrm{H}$ NMR $(\delta \mathrm{ppm})(400 \mathrm{MHz}, \mathrm{DMSO}), \delta 1.1\left(\mathrm{~d}, 6 \mathrm{H}, \mathrm{CH}_{3}\right), \delta 2.1(\mathrm{~m}, 1 \mathrm{H}$, $\mathrm{CH}), \delta 2.8\left(\mathrm{~d}, 2 \mathrm{H}, \mathrm{CH}_{2}\right), \delta 3.3\left(\mathrm{~s}, 3 \mathrm{H}, \mathrm{N}-\mathrm{CH}_{3}\right), \delta$ 7.4-8.4 (dd, $\left.4 \mathrm{H}, \mathrm{Ar}-\mathrm{H}\right), \delta 10.2(\mathrm{~s}, 1 \mathrm{H}, \mathrm{NH})$.

\section{3. 2-(4-Methoxybenzylidenehydrazinyl)-1,6-dihydro-4-isobutyl-1-methyl-6-oxo pyrimidine-5-carbonitrile (4c)}

Mass $\mathrm{M}^{+}=339$ : IR $(\mathrm{KBr})$ v $\left(\mathrm{cm}^{-1}\right), 2954\left(-\mathrm{CH}_{3}\right.$, Asym. $), 2868\left(-\mathrm{CH}_{3}, \mathrm{Sym}.\right), 2220$ ($\mathrm{CN}), 1676(-\mathrm{CO})$; 1H NMR $(\delta \mathrm{ppm})(400 \mathrm{MHz}, \mathrm{DMSO}), \delta 0.9\left(\mathrm{~d}, 6 \mathrm{H}, \mathrm{CH}_{3}\right), \delta 2.1(\mathrm{~m}, 1 \mathrm{H}$, $\mathrm{CH}), \delta 2.3\left(\mathrm{~d}, 2 \mathrm{H}, \mathrm{CH}_{2}\right), \delta 3.1\left(\mathrm{~s}, 3 \mathrm{H}, \mathrm{N}-\mathrm{CH}_{3}\right), \delta 3.3\left(\mathrm{~s}, 3 \mathrm{H},-\mathrm{OCH}_{3}\right), \delta 7.2-7.8(\mathrm{~m}, 4 \mathrm{H}, \mathrm{Ar}-\mathrm{H})$ $\delta 9.8(\mathrm{~s}, 1 \mathrm{H}, \mathrm{NH})$.

\section{4. 2-(4-N,N-dimethyl benzylidenehydrazinyl)-1,6-dihydro-4-isobutyl-1-methyl-6- oxopyrimidine-5-carbonitrile (4d)}

Mass $\mathrm{M}^{+}=352$ : IR $(\mathrm{KBr}) \mathrm{v}\left(\mathrm{cm}^{-1}\right), 2965\left(-\mathrm{CH}_{3}\right.$, Asym.), $2877\left(-\mathrm{CH}_{3}, \mathrm{Sym}.\right), 2218$ ($\mathrm{CN}), 1675$ (-CO); 1H NMR ( $\delta$ ppm) (400MHz, DMSO), $\delta 1.0\left(\mathrm{~d}, 6 \mathrm{H}, \mathrm{CH}_{3}\right), \delta 2.0(\mathrm{~m}, 1 \mathrm{H}$, $\mathrm{CH}), \delta 2.7\left(\mathrm{~d}, 2 \mathrm{H}, \mathrm{CH}_{2}\right), \delta 3.0\left(\mathrm{~s}, 6 \mathrm{H}, \mathrm{CH}_{3}\right) \delta 3.2\left(\mathrm{~s}, 3 \mathrm{H}, \mathrm{N}-\mathrm{CH}_{3}\right), \delta$ 7.4-8.4 (m, 5H, Ar-H) $\delta$ $10.4(\mathrm{~s}, 1 \mathrm{H}, \mathrm{NH})$.

\section{5. 2-(3,4-Dimethoxy benzylidenehydrazinyl)-1,6-dihydro-4-isobutyl-1-methyl-6- oxopyrimidine-5-carbonitrile (4e)}

Mass $\mathrm{M}^{+}=369$ : IR $(\mathrm{KBr})$ v $\left(\mathrm{cm}^{-1}\right), 2956\left(-\mathrm{CH}_{3}\right.$, Asym.), $2868\left(-\mathrm{CH}_{3}, \mathrm{Sym}.\right), 2225$ ($\mathrm{CN}), 1676(-\mathrm{CO}) ; 1 \mathrm{H}$ NMR $(\delta \mathrm{ppm})(400 \mathrm{MHz}, \mathrm{DMSO}), \delta 0.9\left(\mathrm{~d}, 6 \mathrm{H}, \mathrm{CH}_{3}\right), \delta 2.1(\mathrm{~m}, 1 \mathrm{H}$, $\mathrm{CH}), \delta 2.3\left(\mathrm{~d}, 2 \mathrm{H}, \mathrm{CH}_{2}\right), \delta 3.1\left(\mathrm{~s}, 3 \mathrm{H}, \mathrm{N}-\mathrm{CH}_{3}\right), \delta 3.5\left(\mathrm{~s}, 6 \mathrm{H},-\mathrm{OCH}_{3}\right), \delta 7.2-7.8(\mathrm{~m}, 3 \mathrm{H}, \mathrm{Ar}-\mathrm{H})$ $\delta 9.9(\mathrm{~s}, 1 \mathrm{H}, \mathrm{NH})$. 


\section{6. 2-(3-Methoxy- 4-hydroxy benzylidenehydrazinyl)- 1,6-dihydro-4-isobutyl-1-methyl- 6-oxopyrimidine-5-carbonitrile (4f)}

Mass $\mathrm{M}^{+}=355$ : IR $(\mathrm{KBr}) \mathrm{v}\left(\mathrm{cm}^{-1}\right), 2957$ (- $\mathrm{CH}_{3}$, Asym.), 2870 (- $\left.\mathrm{CH}_{3}, \mathrm{Sym}.\right), 2223$ ($\mathrm{CN}), 1670$ (-CO); 1H NMR ( $\delta \mathrm{ppm})(400 \mathrm{MHz}, \mathrm{DMSO}), \delta 0.9(\mathrm{~d}, 6 \mathrm{H}, \mathrm{CH} 3), \delta 2.1(\mathrm{~m}, 1 \mathrm{H}$, $\mathrm{CH}), \delta 2.3(\mathrm{~d}, 2 \mathrm{H}, \mathrm{CH} 2), \delta 3.1\left(\mathrm{~s}, 3 \mathrm{H}, \mathrm{N}-\mathrm{CH}_{3}\right), \delta 3.5\left(\mathrm{~s}, 3 \mathrm{H},-\mathrm{OCH}_{3}\right), \delta$ 7.2-7.8 $(\mathrm{m}, 4 \mathrm{H}, \mathrm{Ar}-\mathrm{H})$, $\delta 9.8(\mathrm{~s}, 1 \mathrm{H}, \mathrm{NH}), \delta 10.1(\mathrm{~s}, 1 \mathrm{H}, \mathrm{OH})$.

\section{7. 2-(4-Methyl benzylidenehydrazinyl)-1,6-dihydro-4-isobutyl-1-methyl-6- oxopyrimidine-5-carbonitrile (4g)}

Mass $\mathrm{M}^{+}=323$ : IR $(\mathrm{KBr})$ v $\left(\mathrm{cm}^{-1}\right), 2972\left(-\mathrm{CH}_{3}\right.$, Asym.), $2860\left(-\mathrm{CH}_{3}, \mathrm{Sym}.\right), 2227$ ($\mathrm{CN}), 1671(-\mathrm{CO}) ; 1 \mathrm{H}$ NMR $(\delta \mathrm{ppm})(400 \mathrm{MHz}, \mathrm{DMSO}), \delta 1.1\left(\mathrm{~d}, 6 \mathrm{H}, \mathrm{CH}_{3}\right), \delta 2.1(\mathrm{~m}, 1 \mathrm{H}$, $\mathrm{CH}), \delta 2.8\left(\mathrm{~d}, 2 \mathrm{H}, \mathrm{CH}_{2}\right), \delta 2.3\left(\mathrm{~s}, 3 \mathrm{H},-\mathrm{CH}_{3}\right), \delta 3.2\left(\mathrm{~s}, 3 \mathrm{H}, \mathrm{N}-\mathrm{CH}_{3}\right), \delta$ 7.4-8.4 (m, 4H, Ar-H), $\delta$ $10.0(\mathrm{~s}, 1 \mathrm{H}, \mathrm{NH})$.

\section{8. 2-(4-Fluro benzylidenehydrazinyl)- 1,6-dihydro-4-isobutyl-1-methyl- 6-oxo pyrimidine- 5-carbonitrile (4h)}

Mass $\mathrm{M}^{+}=327$ : IR $(\mathrm{KBr})$ v $\left(\mathrm{cm}^{-1}\right), 2972\left(-\mathrm{CH}_{3}\right.$, Asym.), $2860\left(-\mathrm{CH}_{3}, \mathrm{Sym}.\right), 2227$ ($\mathrm{CN}), 1671(-\mathrm{CO})$; $1 \mathrm{H}$ NMR $(\delta \mathrm{ppm})(400 \mathrm{MHz}, \mathrm{DMSO}), \delta 1.1\left(\mathrm{~d}, 6 \mathrm{H}, \mathrm{CH}_{3}\right), \delta 2.1(\mathrm{~m}, 1 \mathrm{H}$, $\mathrm{CH}), \delta 2.8\left(\mathrm{~d}, 2 \mathrm{H}, \mathrm{CH}_{2}\right), \delta 3.2\left(\mathrm{~s}, 3 \mathrm{H}, \mathrm{N}-\mathrm{CH}_{3}\right), \delta$ 7.4-8.2 (m, 4H, Ar-H), $\delta 10.0(\mathrm{~s}, 1 \mathrm{H}, \mathrm{NH})$.

\section{9. 2-(4-Hydroxy benzylidenehydrazinyl)-1,6-dihydro-4-isobutyl-1-methyl-6- oxopyrimidine-5-carbonitrile (4i)}

Mass $\mathrm{M}^{+}=325$ : IR $(\mathrm{KBr}) \mathrm{v}\left(\mathrm{cm}^{-1}\right), 2955\left(-\mathrm{CH}_{3}\right.$, Asym.), $2874\left(-\mathrm{CH}_{3}, \mathrm{Sym}.\right), 2220$ ($\mathrm{CN}), 1678(-\mathrm{CO}) ; 1 \mathrm{H}$ NMR $(\delta \mathrm{ppm})(400 \mathrm{MHz}, \mathrm{DMSO}), \delta 0.9(\mathrm{~d}, 6 \mathrm{H}, \mathrm{CH} 3), \delta 2.1(\mathrm{~m}, 1 \mathrm{H}$, $\mathrm{CH}), \delta 2.3\left(\mathrm{~d}, 2 \mathrm{H}, \mathrm{CH}_{2}\right), \delta 3.1\left(\mathrm{~s}, 3 \mathrm{H}, \mathrm{N}-\mathrm{CH}_{3}\right), \delta 7.2-7.8(\mathrm{~m}, 4 \mathrm{H}, \mathrm{Ar}-\mathrm{H}), \delta 9.8(\mathrm{~s}, 1 \mathrm{H}, \mathrm{NH}), \delta$ $10.1(\mathrm{~s}, 1 \mathrm{H}, \mathrm{OH})$.

\section{10. 2-(2-Chloro benzylidenehydrazinyl)-1,6-dihydro-4-isobutyl-1-methyl-6- oxopyrimidine-5-carbonitrile $(4 \mathbf{j})$}

Mass $\mathrm{M}^{+}=343$ : IR $(\mathrm{KBr})$ v $\left(\mathrm{cm}^{-1}\right), 2963\left(-\mathrm{CH}_{3}\right.$, Asym.), $2876\left(-\mathrm{CH}_{3}, \mathrm{Sym}.\right), 2225$ ($\mathrm{CN}), 1668(-\mathrm{CO}) ; 1 \mathrm{H}$ NMR $(\delta \mathrm{ppm})(400 \mathrm{MHz}, \mathrm{DMSO}), \delta 1.1\left(\mathrm{~d}, 6 \mathrm{H}, \mathrm{CH}_{3}\right), \delta 2.1(\mathrm{~m}, 1 \mathrm{H}$, $\mathrm{CH}), \delta 2.8\left(\mathrm{~d}, 2 \mathrm{H}, \mathrm{CH}_{2}\right), \delta 3.3\left(\mathrm{~s}, 3 \mathrm{H}, \mathrm{N}-\mathrm{CH}_{3}\right), \delta$ 7.4-8.4 (m, $\left.4 \mathrm{H}, \mathrm{Ar}-\mathrm{H}\right), \delta 10.2(\mathrm{~s}, 1 \mathrm{H}, \mathrm{NH})$.

\section{CONCLUSION}

Rarely reported Benzylidinehydrazinyl derivatives of 4-alkyl pyrimidine-5-carbonitrile were prepared using 2-hydrazinyl-1,6-dihydro-1-methyl-6-oxo-4-isobutylpyrimidine-5carbonitrile (3) with different aromatic aldehyde in presence of 1-2 $\mathrm{ml}$ of glacial acetic acid gave 2-(Substituted benzylidenehydrazinyl)-1,6-dihydro-4-isobutyl-1-methyl-6oxopyrimidine-5-carbonitrile (4a-j) (Scheme 1). All synthesized compounds were obtained in good yield (Table 1). The synthesized compounds were characterized by IR, ${ }^{1} \mathrm{H}-\mathrm{NMR}$ and Mass Spectroscopy and the obtained results are showing good agreement with the synthesized structure. 


\section{ACKNOWLEDGEMENT}

Authors are thankful to Maharshi Dayanand Science College, Porbandar for providing research facilities. We are grateful the NFDD center, Saurashtra University, Rajkot for recording and providing ${ }^{1} \mathrm{H}$ NMR, Mass and IR Spectral data.

\section{References}

[1] H. Schiff, Ann. Chem. 343(1864), 3.

[2] D.N. Dhar, C.L. Taploo, J Sci Ind Res 41 (1982) 501-506.

[3] P. Przybylski, A. Huczyński, K. Pyta, B. Brzezinski, F. Bartl, Curr Org Chem 13 (2009) 124-148.

[4] G. Bringmann, M. Dreyer, J.H. Faber, P.W. Dalsgaard, D. Staerk, J.W. Jaroszewski, J Nat Prod 67(5) (2004) 743-748.

[5] J. Salimon, N. Salih, H. Ibraheem, E. Yousif, Asian J Chem 22(7) (2010) 5289-5296.

[6] Z. Guo, R. Xing, S. Liu, Z. Zhong, X. Ji, Wang L., 342(10) (2007) 1329-1332.

[7] Y. Li, Z.S. Yang, H. Zhang, B.J. Cao, F.D. Wang, Bioorg, Med Chem 11 (2003) 4363-4368.

[8] J.J. Modha, J.M. Parmar,N.J. Datta, H.H. Parekh, Indian J. Chem. B 41B(12) (2002) 2694-2697.

[9] J.J. Modha, N.J. Datta, H.H. Parekh, Il Farmaco 56 (2001) 641-646.

[10] Cho Hidetsura, Ueda Masaru, Eur. Pat. Appl. EP 280,227(Cl. C07D 239/20), 31 Aug 1988 JP Apply 87/39,345,21 Feb. 198720 pp.

[11] Atwal Karnial, U.S.4, 769,371(Cl,514-275;C07D 239/42)6th Sept.1988.Appl 4, 595601 May 198714 pp.

[12] Ueda Taisei, Sakkakibara Jinsaku, Nakagami Jozi, Chem Pharma Bull 31(12) (1983) 4263-4269.

[13] Imperial Chemical Industry Ltd. Brit. Appl. 3 (1959) 876, 601.

[14] Shakhidoyatov K. M., et al. Ch. Sh (USSR) Fungitsidy 1980 66-81 (Russ) Edited by Melnikov N N, Izd Fan Uzb SSR Tashkent USSR.

[15] El-Zohry F. Maher, Abd Alla Mohammad, Chem. Technol. Biotechnol 55(3) (1992) 15-20.

[16] Taggart M. T., et al. Curv Chemother Infect. Dist. Proc. Int. Congr. Chemother 11th 1979 Pub. (1980)2, 1400-1 Eng. Edited by nelson John D.

[17] S. Mohammad, S. Ahmad Khan, A. Mohammad, M.A. Mohammad, Saudi Pharm. J. 20 (2012) 149-154. 
[18] Taghrid S. Hafez, Souad A. Osman, Hisham Abdallah, A. Yosef et al., Sci.pharm. 10 (2013) 1211-07.

[19] A. K. Gupta, S. Saini, R. Pal, R. Kumar, V. Beniwal, World J. of Pharmacy and Pharma. Sci. 3(8) (2014) 1621-1636.

[20] Kambe Satoshi, Saito Koji, Kishi Hiroshi, Sakurai Akio, Midorikawa Hiroshi, Oyama Tech. Coll. Tochigi, Japan 4 (1979) 287-289. 